\title{
Time Dependent Effect of flow properties on addition of heat to the air flow at Compressible Subsonic Speeds
}

\author{
Balaguru Pandian ${ }^{1}$, Rajarshi Das ${ }^{2, *}$ and Heuy Dong Kim ${ }^{3}$ \\ 1,2, *Gas Dynamics and Propulsion Labaratory, School of Mechanical Engineering, SASTRA \\ Deemed to be University, Thanjavur 613401, India \\ ${ }^{3}$ Gas Dynamics Lab, Department of Mechanical Engineering, Andong National University, \\ Andong 760749, Republic of Korea \\ ${ }^{1}$ Email: balaguru@sastra.ac.in \\ 2, *Email: rajarshidas@mech.sastra.edu \\ ${ }^{3}$ Email: kimhd@andong.ac.kr
}

\begin{abstract}
Heat addition process is a governing phenomenon in turbojet and ramjet burners, where heat is added to the flow during the fuel-air combustion process. In the present work, an analytical study has been performed to investigate the effect of heat addition on the basic flow properties based on compressible Rayleigh flow model between the subsonic and sonic flow regime. The unsteady nature of flow properties in these regimes have been evaluated analytically by adding heat through the walls of a constant area, frictionless duct in which air flow occurs over different periods of time. Sudden variation of temperature, pressure, velocity and kinetic energy of the flow were found at flow Mach number $M=0.94$. Flow velocity and kinetic energy increases until the flow approaches $M=0.94$, afterwards there is a rapid increase in the flow velocity and kinetic energy until it reaches sonic conditions. Conversely, pressure decreased till the flow reaches $M=0.94$, there after a sudden drop was observed. Flow enthalpy increased initially, reached maximum at flow $M=0.845$ and then reduced till sonic conditions. Based on the observations, it has been concluded that the heat energy contributed to increase the kinetic energy thereby reducing the temperature of the flow.
\end{abstract}

Keywords: Compressible flow, Heat addition, Temperature inversion, Unsteady calculations, Mach number

\section{Introduction}

Rayleigh flow heat addition process is a governing phenomenon in all combustors, where heat is added to the flow during the fuel-air combustion process. This theory is based on the steady and inviscid assumptions of diabatic compressible flow of gas through a straight conduit and has long been applied to the thermal choking problems in numerous miscellaneous engineering fields. However, this theory has seldom been authenticated with experimental data. Time-dependent behaviour of the Rayleigh flow has rarely been investigated so far. However, in compressible flows, the heat addition process is fundamentally unsteady in nature with pressure time variations which can be connected to wave motions. This paper emphasizes on the effect of heat transfer on basic flow properties in a constant area flow channel, for a compressible, inviscid flow. The pertinence of this kind of study is to investigate the nature of flow in compressible subsonic and transonic combustors and other applications where heat is transferred in between these regimes. Studies have been conducted so far to examine this phenomenon in the vicinity of sonic conditions, when the flow gets thermally choked [1-3]. Guo [4] uncovered the unconventional concept of thermal clogging during heat addition process for sub-sonic flow in duct. The main focus was to boost the researchers to develop supersonic combustors with higher flow M. It was revealed rapidly that, for a flow with $\gamma$ $=1.4$, in the range of $0.845 \leq \mathrm{M} \leq 1.0$, there occur an inversion of thermal effect of heat addition [5].

Zczeniowski [6] analytically investigated the flow of gas through a constant area duct with heat exchange through the tube walls and concluded that, the heat exchange was not possible when the flow attains Mach number of 1. Separate studies conducted by Kahane and Lees [7] concluded that when the heat was suddenly added to flow in tubes of varying cross-sectional areas, there was substantial adjustments of upstream conditions of the

*Senior Assistant Professor, Gas Dynamics and Propulsion Laboratory, School of Mechanical Engineering, SASTRA Deemed to be University, Thanjavur 613401, India: Email:

rajarshidas@mech.sastra.edu (Corresponding author) 
flow. Shapiro discussed in detail about the interdependence of basic flow parameters in similar flows under various flow conditions [1]. The process of heat addition to compressible flow has been described for steady conditions by relating dependent and independent variables with the influence parameters. It has been noted that, existence of steady-state flow is not possible during choking, and after a certain period of transient readjustment, a new updated steady-state condition is attained for which choking does not occur. Studies on non-equilibrium thermodynamics of an electron gas in Rayleigh flow using unsteady Boltzmann equation revealed that the effect of the changes of the internal energies due to variation of polarization, induced magnetic in-duction and magnetization due to electric and magnetic fields were very small when it is compared with the internal energy change due to variation in entropy [8]. Similar studies have been conducted in molecular level by adding heat in to compressible flows to investigate the electron gas particle interaction in such flows [9] and claim of such development for nozzle flows under steady heat transfer process [10]. Das and Kim [12] analytically investigated the unsteady Rayleigh flow in the vicinity of choking conditions based on fundamental governing equations. They found that there was a variation of flow Mach number on continuous unsteady heating through the choking point. From the obtained results, they concluded that the effect of mass flow rate cannot be ignored at the choking point for both subsonic and super-sonic Rayleigh flows.

Till now, there has been no conclusive explanation available for the temperature inversion effect between $\mathrm{M}=0.845(\mathrm{M}=1 / \sqrt{ } \gamma)$ and $\mathrm{M}=1$ when heat is added to subsonic compressible flow of air. Existing literature have not explained clearly about the physical phenomena occurring during such transition. Recent works have mainly focused on transition of flows in and around the choking point when heat is transferred to it [12]. For development of subsonic and supersonic flow combustor, investigation into such flows in the vicinity of $\mathrm{M}=1$ is required. Propulsive efficiency of the combustor can be affected due to combustion instability in case of any unknown phenomenon occurring at such speeds.

The addition on heat to compressible flows is theoretically explained by the Rayleigh flow phenomena as shown by the Rayleigh curve in Figure 1.1. Represented on an enthalpy (temperature)-entropy plot the Rayleigh curve shows an increase of flow temperature till $\mathrm{M}=0.845$ for air. Therefore, present study has tried to investigate the unsteady nature of flow properties occurring in the region of $0.7 \leq \mathrm{M} \leq 1.0$ by adding heat through the walls of the constant area, frictionless duct.

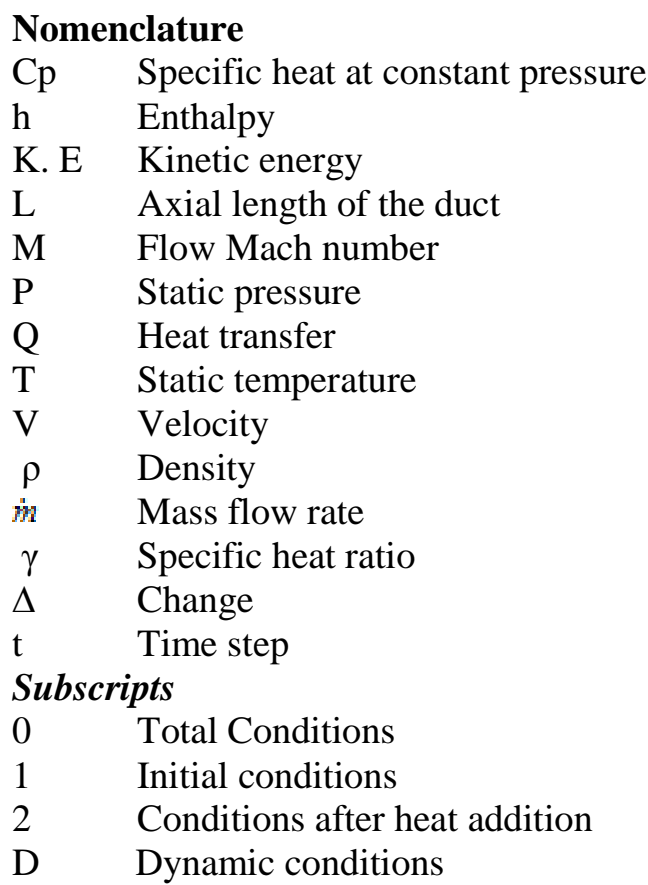




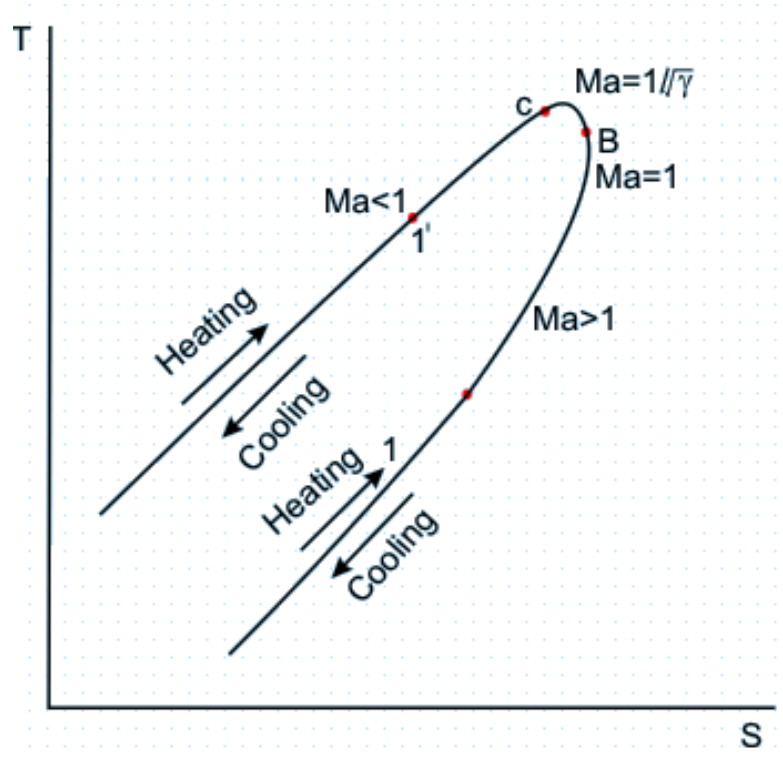

Figure 1.1 Rayleigh curve

\section{Governing Equations and Solution Methodology}

Let us assume that a one-dimensional flow occurs through a control surface as shown in Figure 2.1. Air flows through the control surface with an initial temperature of $273.224 \mathrm{~K}$, atmospheric pressure of $101325 \mathrm{~Pa}$ and Mach number of $\mathrm{M}=0.7$. The initial total temperature was found to be at $300 \mathrm{~K}$ and initial density was $0.9697 \mathrm{~kg} / \mathrm{m} 3$. The temperature outside the duct was assumed to be at $1500 \mathrm{~K}$ while the temperature inside the duct was at $300 \mathrm{~K}$. Let us consider that the air flows through the stainless-steel duct with diameter of $1 \mathrm{~m}$ and wall thickness of $10 \mathrm{~mm}$ and heat dQ was transferred through the walls of the duct. Eq. (1) gives the amount of heat transferred through the duct wall which is modelled as a function of time and wall thickness. The temperature change of the inner wall of the duct depends on the heat transfer and it is governed by Eq. (2). Based on the one-dimensional heat equation, the heat conduction through the wall is modelled as shown in Eq. (3). Eq. (3) is solved using numerical integration by taking the initial conditions into consideration.

$$
\mathrm{Q}=\mathrm{m} \Delta \mathrm{T}(\mathrm{L}, \mathrm{t}) \mathrm{C}_{\mathrm{p}}
$$

$\Delta \mathrm{T}(\mathrm{x}, \mathrm{t})=\sum_{\mathrm{n}=1}^{\infty} \sin \left(\frac{\mathrm{nmx}}{\mathrm{I}}\right) \mathrm{C}_{\mathrm{n}} \mathrm{e}^{-\left(\frac{\mathrm{A}^{2} \mathrm{x}^{2} \mathrm{~s}}{\mathrm{I}^{2}}\right)}$

where

$$
\mathrm{C}_{\mathrm{n}}=\frac{2}{\mathrm{~L}} \int_{0}^{\mathrm{L}} \Delta \mathrm{T}(\mathrm{x}, 0) \sin \frac{\mathrm{nnx}}{\mathrm{L}} \mathrm{dx}
$$

$\frac{\partial T}{\partial x^{2}}=\frac{a T}{a \partial t}$

$\mathrm{T}_{01}=\mathrm{T}_{1}\left(1+\frac{\left.\mathrm{Wp}^{-1}-1\right) \mathrm{M}_{1}^{2}}{2}\right)$

$\mathrm{T}_{02}=\frac{\mathrm{dQ}}{\mathrm{C}_{\mathrm{p}}}+\mathrm{T}_{01}$ 


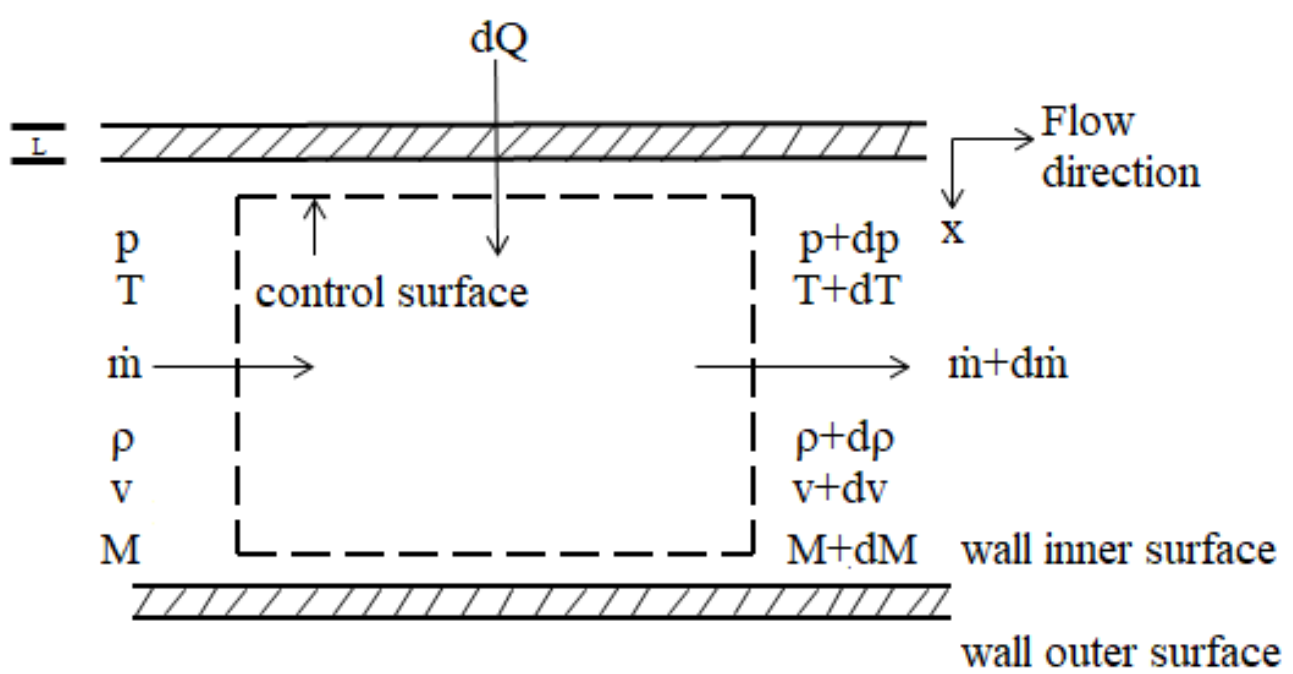

Figure 2.1 Schematic of control surface. [12]

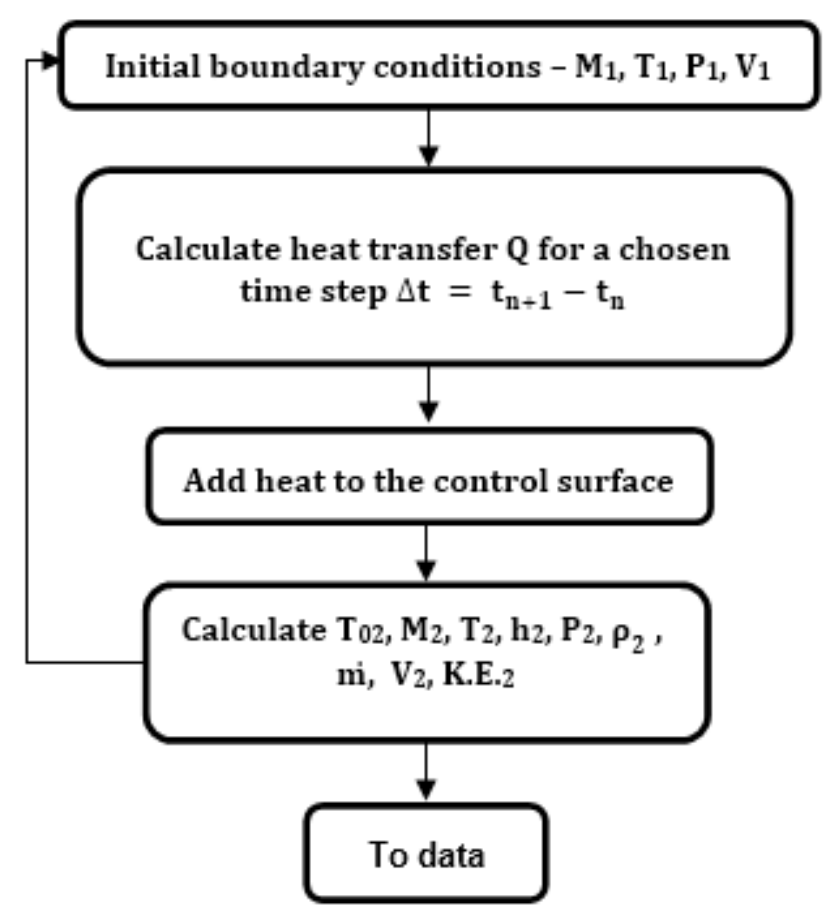

Figure 2.2 Flow chart for methodology adopted for solution

$\mathrm{T}_{01}=\mathrm{T}_{1}\left(1+\frac{(\mathrm{T}-1) \mathrm{N}_{1}^{2}}{2}\right)$

$\mathrm{T}_{02}=\frac{\mathrm{dQ}}{\mathrm{c}_{\mathrm{p}}}+\mathrm{T}_{01}$

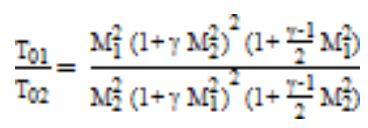

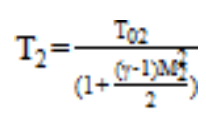

$\mathrm{h}_{2}=\mathrm{C}_{\mathrm{p}} \mathrm{T}_{2}$ 


$$
\begin{aligned}
& \mathbf{P}_{2}=\mathbf{P}_{1} \frac{\left(1+\gamma \mathbf{N}_{1}^{2}\right)}{\left(1+\gamma \mathbf{N}^{2}\right)} \\
& \mathrm{v}_{2}=\mathrm{M}_{2} \sqrt{\gamma \mathrm{RT}_{2}} \\
& P_{2}=P_{1} \frac{M_{1}^{2}\left(1+p_{1}^{2}\right)}{N_{2}^{2}\left(1+7 \mathbf{N}_{1}^{2}\right)} \\
& \mathrm{m}=\mathrm{p}_{2} \mathrm{v}_{2} \\
& \mathrm{KE}_{-2}=\frac{1}{2} \mathrm{mv}_{2}^{2}
\end{aligned}
$$

Heat transfer dQ is evaluated for time step with increment of $\Delta t=10^{-08} \mathrm{~S}$ [12]. This particular time step was chosen because, upon investigation, it is found that when the time step gets smaller, smooth variation in the flow properties are observed instead of irregular variation detected in the respective curves for the time step of $\Delta t=10^{-07} \mathrm{~s}$. This is elaborated in the later part of this paper based on discussion regarding Figure 4. The initial total temperature is a function of flow static temperature and Mach number which was calculated by using compressible flow relations as shown in Eq. (4). By using Eq. (5) the change in total temperature for each time step was evaluated. Based on change in total flow temperature, the flow Mach number $\mathrm{M}_{2}$ was determined by using implicit equation, Eq. (6). In these equations, subscripts " 1 " and " 2 " represents initial conditions and conditions after heat addition in the corresponding time step increments respectively.

The static temperature and enthalpy $[1,11]$ were calculated using the Eq. (7) and Eq. (8). The values obtained at each time step were fed as the initial value for the next time step. The same procedure was followed until the flow gets choked i.e., Mach number $\mathbf{M}_{2}=1.0$. Pressure, density, velocity, mass flow rate and kinetic energy were calculated for the incremental time step using one dimensional fluid flow relations [1,11], Eq. (9) to Eq. (13). The property variations were evaluated until the flow attains Mach number of $\mathrm{M}=$ 1.0. The flow chart for the methodology adopted for theoretical analysis of Rayleigh flow in this work is shown in Figure 2.2.

\section{Results and discussion}

The present study aims at investigating the time dependent flow property variations in the range of $0.7 \leq \mathrm{M} \leq 1.0$ and to access the reasons pertaining to the temperature inversion effect. A constant area, frictionless duct has been used to examine the unsteady nature of flow properties by adding heat through the walls of the duct. Changes in flow properties over different period of time and flow Mach number has been probed during the investigation and the variation of flow properties are correlated to explain the temperature inversion effect.

\subsection{Effect of time step size on enthalpy variation ratio with flow Mach number}

Figure 3.1 and Figure 3.2 shows the enthalpy variation ratio with flow Mach number for different time step sizes such as $\Delta \mathrm{t}=10^{-07} \mathrm{~s}$ and $\Delta \mathrm{t}=10^{-08} \mathrm{~s}$ respectively. For both time step size, it is observed that the enthalpy increases from Mach number 0.7 to 0.845 and decreases beyond it, even though the heat is transferred through the walls of the duct to the flow. Also, as observed in Figure 3.1 and Figure 3.2, for the time step size $\Delta t=10^{-07} \mathrm{~s}$, the enthalpy variation shows a point of inflection just beyond $\mathrm{M}=0.84$. Conversely, smooth variation of enthalpy is observed for the time step $\Delta t=10^{-08}$ s. Hence, time step increment of $\Delta \mathrm{t}=10^{-08} \mathrm{~s}$ is used for further theoretical analysis. 


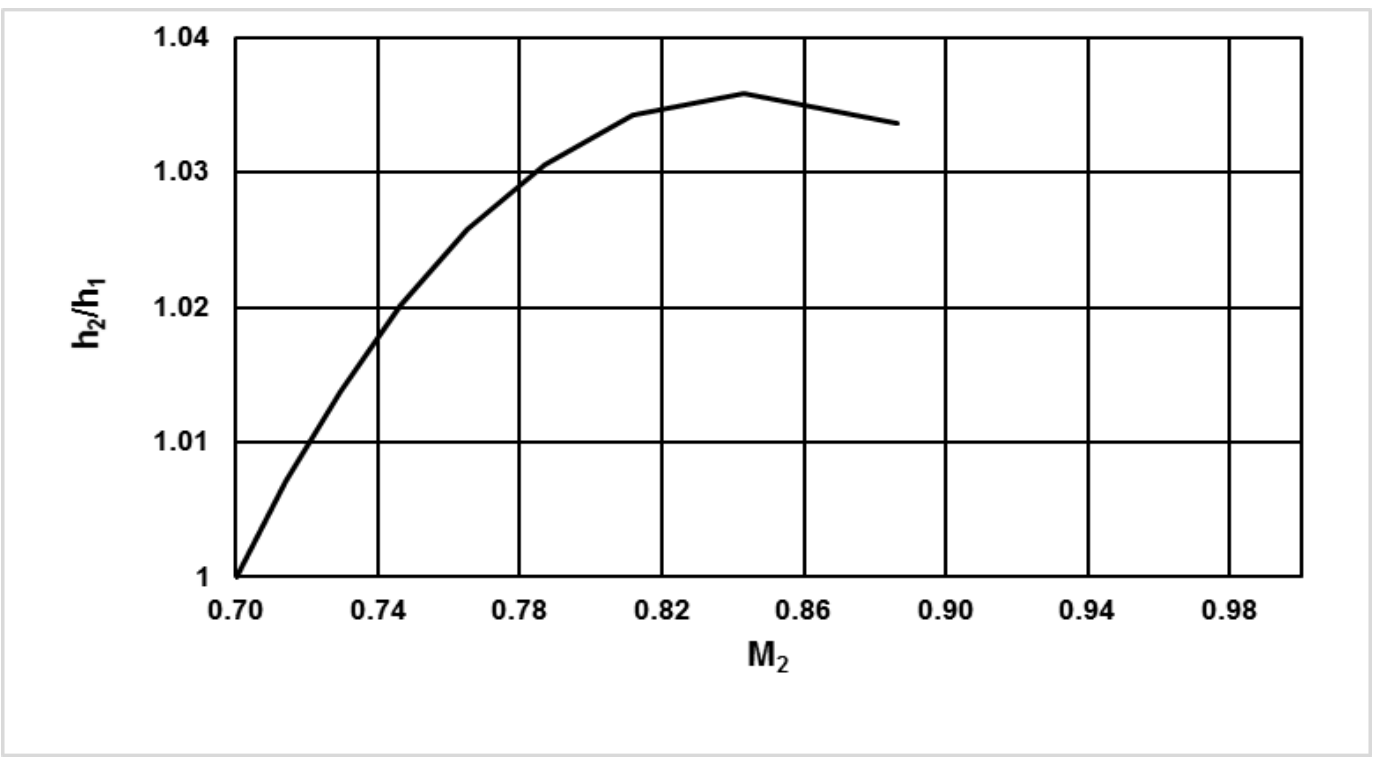

Figure 3.1. Variation of enthalpy with flow Mach number for time step size $\Delta \mathrm{t}=10^{-07} \mathrm{~s}$

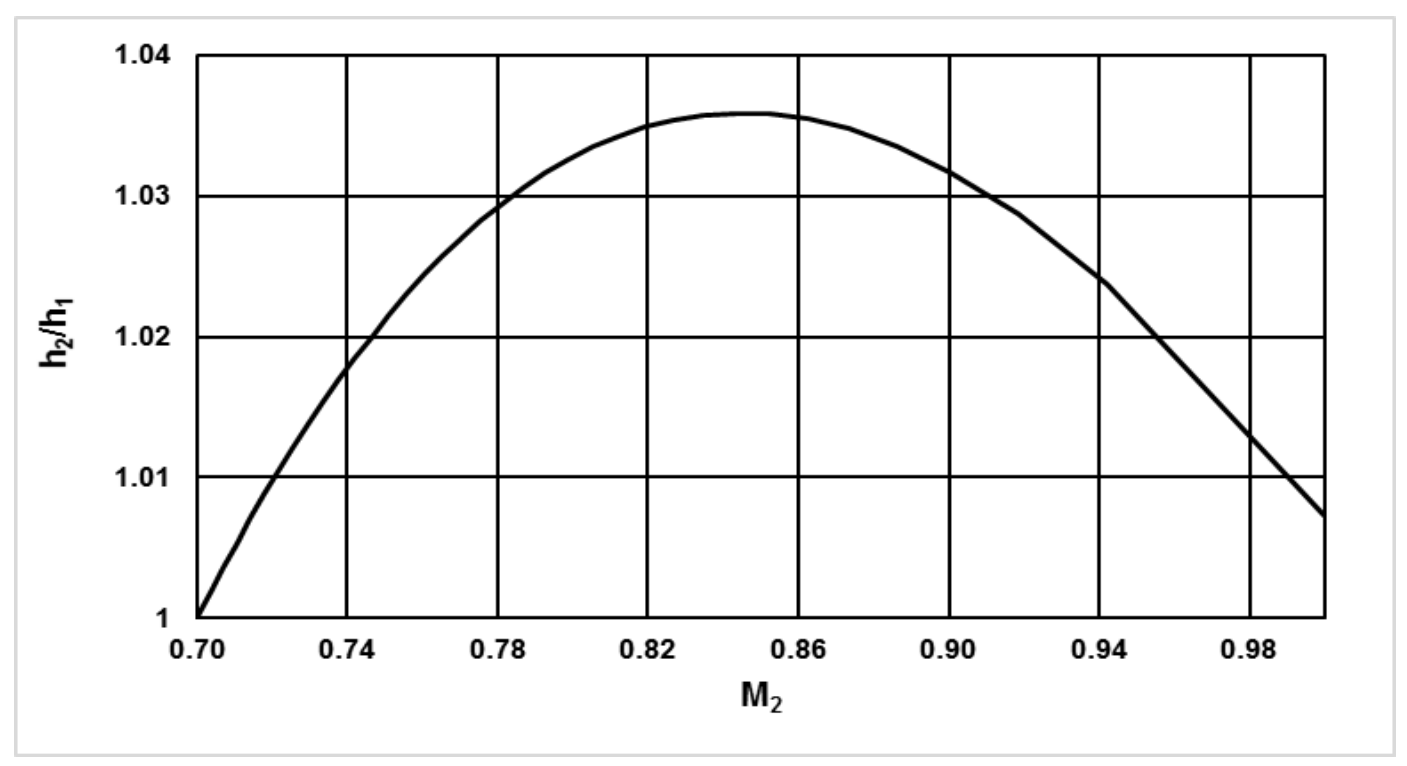

Figure 3.2. Variation of enthalpy with flow $M$ for time step size $\Delta t=10^{-08} \mathrm{~S}$

\subsection{Effect of flow properties with flow Mach number}

Figure 3.3 shows the variation of the evaluated flow Mach number with time step of equal intervals of $\Delta t=2.5 \times 10^{-08} \mathrm{~s}$. It has been found that the flow Mach number increases slowly from $\mathrm{M}=0.7$ which is assumed as our initial condition for this investigation, until it reaches $\mathrm{M}=0.94$. Then, there is a rapid increase in the flow Mach number from $M=$ 0.94 to the sonic conditions for the same time interval $\Delta \mathrm{t}=2.5 \times 10^{-08} \mathrm{~s}$. Calculations from change in flow Mach number shows that $\Delta \mathrm{M}_{2}=0.06$ between $\mathrm{M}=0.94$ and sonic state, which is 3 times greater when compared to the previous time steps change in flow Mach number, that is $\Delta \mathrm{M}_{2}=0.02$.

The change in flow Mach number has been considered as the major parameter among all other flow parameters, to determine the other flow properties such as temperature, pressure, velocity and kinetic energy. In order to investigate the variation in flow properties between compressible subsonic and sonic flow regime, all these flow 
parameters have been non-dimensionalized with its initial values respectively. Hence, in this section, flow parameters variation with flow Mach number $\mathrm{M}$ between $\mathrm{M}=0.7$ to $\mathrm{M}$ $=1$ has been presented and discussed. Variation of temperature with flow $\mathrm{M}$ shows similar variation as enthalpy as is observed in Figure 3.2 and Figure 3.4.

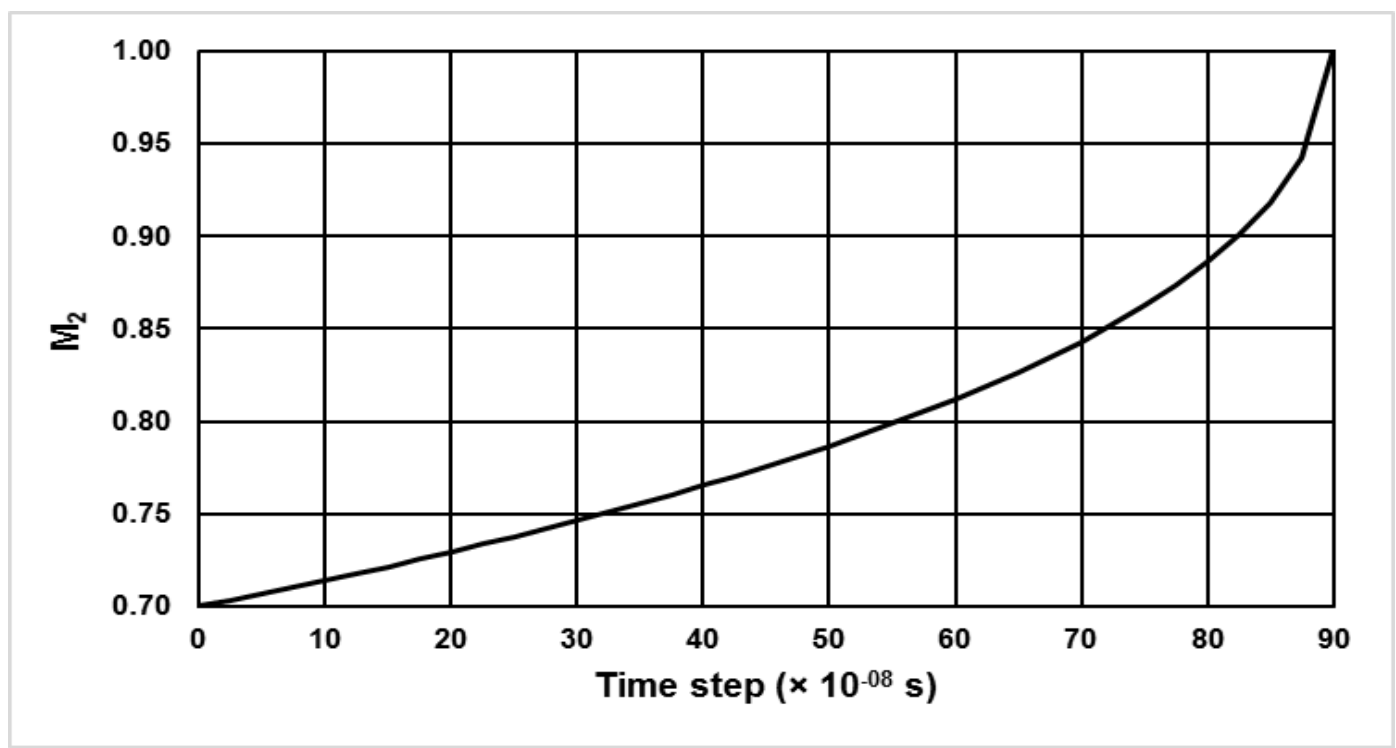

Figure 3.3 Variation of flow Mach number with time step

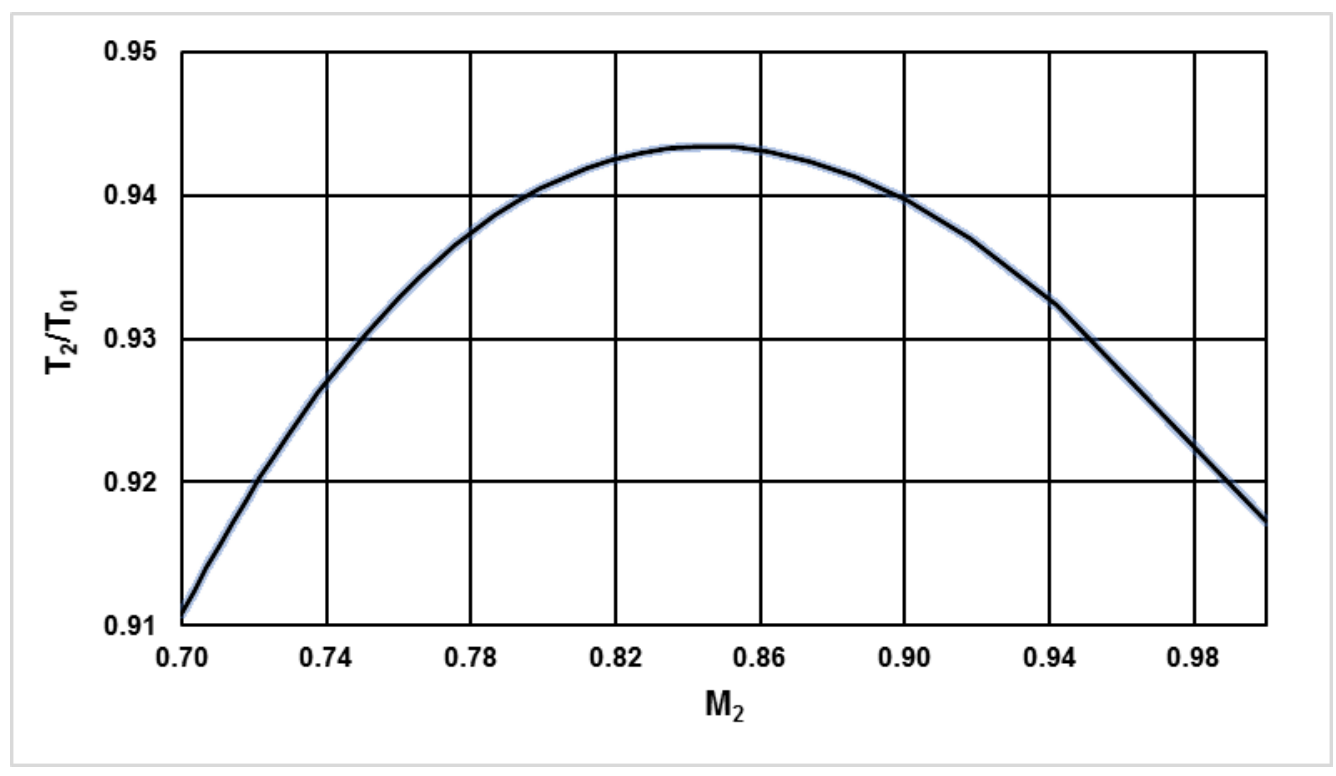

Figure 3.4 Variation of temperature with flow Mach number

Initially, the temperature increases, but after the flow reaches $M=0.845$, the temperature decreases. Once, it reaches Mach 0.94, there is a sudden decrease in temperature change. The change expressed as $\Delta\left(\mathrm{T}_{2} / \mathrm{T}_{01}\right)$ is observed to be 0.0046 between $\mathrm{M}=0.92$ to $\mathrm{M}=$ 0.94 and jumps to a value of 0.015 between $\mathrm{M}=0.94$ to $\mathrm{M}=1.0$. Further it is observed that between $\mathrm{M}=0.85$ to $\mathrm{M}=0.94, \Delta\left(\mathrm{T}_{2} / \mathrm{T}_{01}\right)$ has an average value of 0.0016 , which is almost 9.5 times lesser in the magnitude for the same time step increment captured between $\mathrm{M}=0.94$ to $\mathrm{M}=1.0$. This leads to a sudden decrease in temperature being observed, when the flow approaches sonic conditions when compared to previous temperature decrement for the same time interval. The maximum temperature ratio $T_{2} / T_{01}$ reaches at flow Mach number 0.845 as 0.9434. 
Pressure variation $\mathrm{P}_{2} / \mathrm{P}_{1}$ with flow Mach number $\mathrm{M}$ has been presented in Figure 3.5. From the plot, it has been observed that there is a continuous decrement in pressure ratio from $M=0.7$ to $M=1$. Pressure ratio change has been identified for three different $\Delta M_{2}$ such as from $M=0.7$ to $M=0.845$, from $M=0.845$ to $M=0.94$ and from $M=0.94$ to $M=1$. It has been observed that the change in pressure ratio $\Delta\left(\mathrm{P}_{2} / \mathrm{P}_{1}\right)$ is decreases between $\mathrm{M}=0.7$ to $\mathrm{M}=0.845$. Afterwards, an increase in the pressure decrement is observed between $\mathrm{M}=$ 0.845 to $\mathrm{M}=0.94$ and then there is a sudden sharp increase in pressure decrement between $M=0.94$ to $M=1$. The average change in pressure ratio $\Delta\left(\mathrm{P}_{2} / \mathrm{P}_{1}\right)$ values has been calculated as 0.0055 and 0.0133 for first two $\Delta \mathrm{M}_{2}$ respectively. From the calculations, it has been observed that the average change in pressure decrement ratio is almost 2.4 times greater for $0.845<\mathrm{M}<0.94$ when compared to $0.7<\mathrm{M}<0.845$. Between $\mathrm{M}=0.94$ and $\mathrm{M}=1, \Delta\left(\mathrm{P}_{2} / \mathrm{P}_{1}\right)=0.0493$, which is almost 2.3 times greater in magnitude for the same time step interval (i.e., between $\mathrm{M}=0.92$ and $\mathrm{M}=0.94$ ) and 3.7 times greater in magnitude when compared to the average value of $\Delta\left(\mathrm{P}_{2} / \mathrm{P}_{1}\right)$ between $\mathrm{M}=0.845$ and $\mathrm{M}=$ 0.94 . From the obtained results, it can be clearly seen that from the pressure ratio - flow Mach number plot, pressure decreases rapidly, once the flow approaches the sonic conditions which means that, there is low pressure in the flow field near the vicinity of sonic state. The minimum value of pressure ratio $P_{2} / P_{1}$ has been found as 0.7026 at flow Mach number 1.

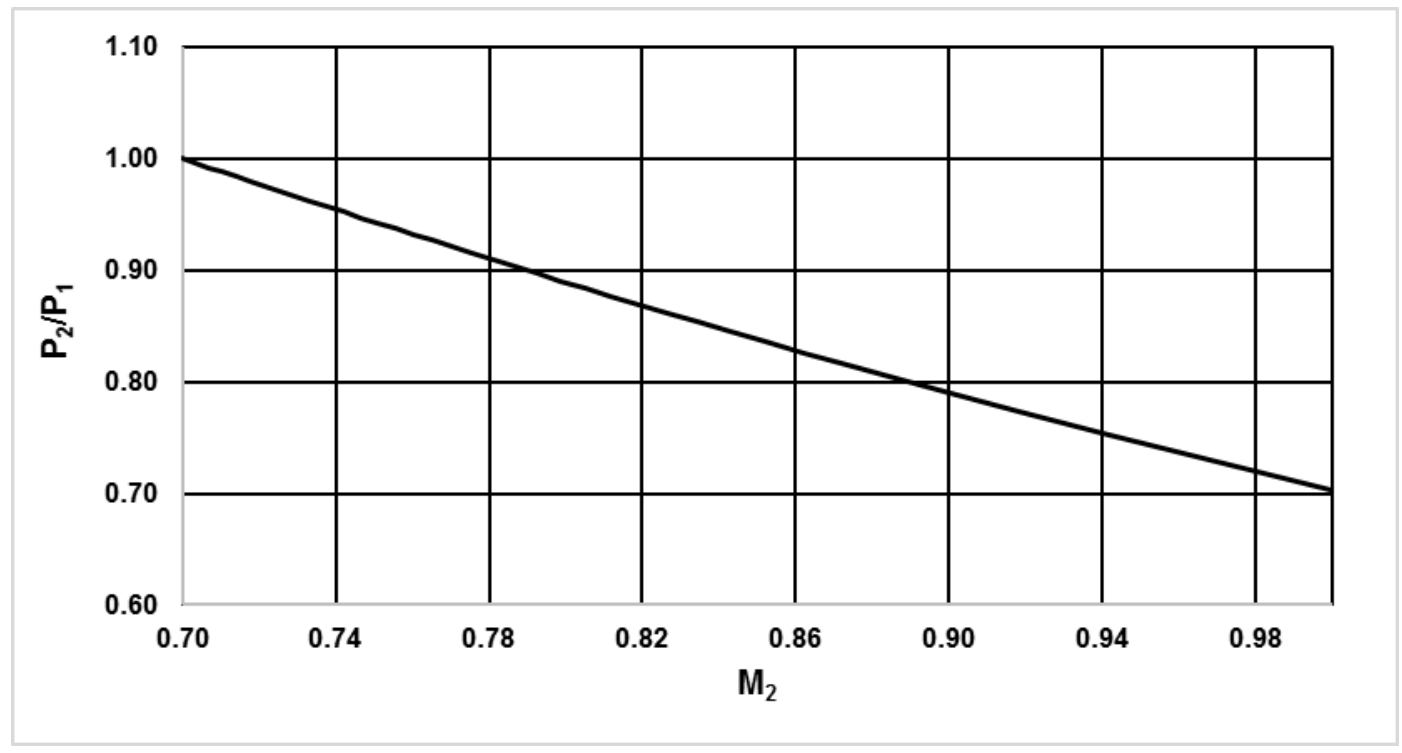

Figure 3.5. Variation of pressure with flow Mach number

The variation of velocity ratio $V_{2} / V_{1}$ with flow Mach number $M$ is presented in Figure 3.6. As compared to pressure change, the velocity ratio varied conversely. From Figure 3.6 , it is observed that the velocity ratio increases steeply between $\mathrm{M}=0.7$ to $\mathrm{M}=1$. Change in velocity ratio $\Delta\left(\mathrm{V}_{2} / \mathrm{V}_{1}\right)$ for three different $\Delta \mathrm{M}_{2}$ is calculated similar to $\Delta \mathrm{P}_{2} / \mathrm{P}_{1}$ variation. The average value velocity ratio change $\Delta\left(\mathrm{V}_{2} / \mathrm{V}_{1}\right)$ for first two $\Delta \mathrm{M}_{2}$ is 0.0081 and 0.0194 respectively. It is also observed that the average change in velocity increment ratio for $0.845<\mathrm{M}<0.94$ is 2.4 times greater in magnitude when compared to the average change in velocity increment ratio for $0.7<\mathrm{M}<0.845$. Also, it is found that the change in velocity ratio value between $\mathrm{M}=0.94$ to $\mathrm{M}=1$ as $\Delta\left(\mathrm{V}_{2} / \mathrm{V}_{1}\right)=0.0719$, which is almost 2.3 times greater in magnitude for the same time step interval used in previous $\Delta \mathrm{M}_{2}$ and 3.7 times greater in magnitude when compared to the average value of $\Delta\left(\mathrm{V}_{2} / \mathrm{V}_{1}\right)$ for $\mathrm{M}=0.845$ to $\mathrm{M}=0.94$ respectively. The obtained results show that velocity ratio suddenly increases once the flow approaches $\mathrm{M}=0.94$, which means near the immediate 
vicinity of the sonic state there is a very high velocity increment as compared to lower $\mathrm{M}$. The maximum velocity ratio $\mathrm{V}_{2} / \mathrm{V}_{1}$ value has been found as 1.4337 at sonic state.

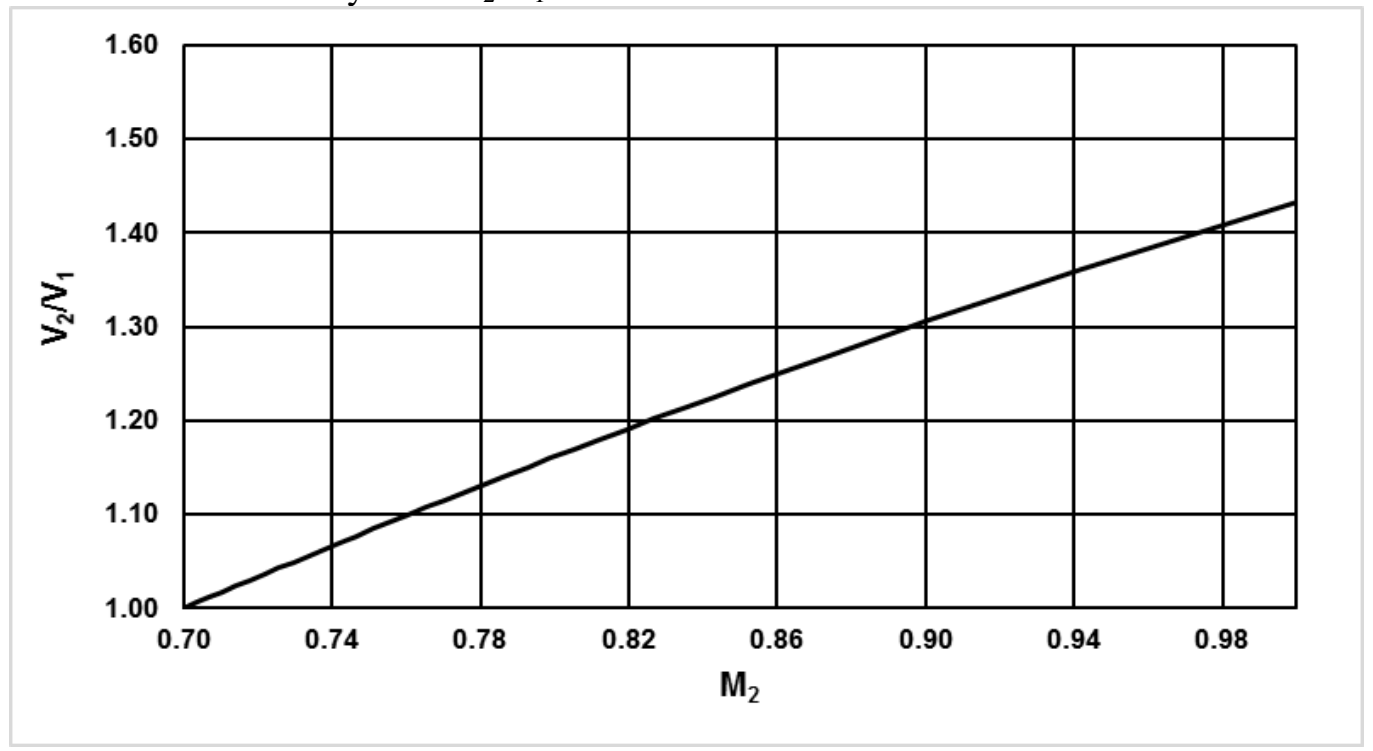

Figure 3.6. Variation of velocity with flow Mach number

Figure 3.7 shows the variation of kinetic energy ratio K.E $E_{2} / K_{1} E_{1}$ with flow Mach number $\mathrm{M}$. Since kinetic energy is directly proportional to the mass flow rate and square of the flow velocity, the kinetic energy ratio trendline is similar to the velocity ratio curve. From the K.E $E_{2} / K . E_{1}$ vs $M$ curve, it has been observed that the kinetic energy sharply increases between flow $M=0.7$ to $M=1$. Similar observations have been made for kinetic energy ratio curve in tune with the other flow properties curves and the results revealed the average change in kinetic energy ratio $\Delta\left(K . E_{2} / K . E_{1}\right)$ for three different $\Delta \mathrm{M}_{2}$ regime. For the first regime, when $0.7<\mathrm{M}<0.845$, the kinetic energy ratio increases with a low gradient and then for second regime between $0.845<\mathrm{M}<0.94$, the gradient increased. Finally, for $0.94<M<1$, the kinetic energy ratio increases with a high gradient. The average change in kinetic energy ratio $\Delta\left(\mathrm{K} \mathrm{E}_{2} / \mathrm{K} . \mathrm{E}_{1}\right)$ values for the first two regimes are 0.0180 and 0.0503 respectively. From these values, it has been observed that the average change in kinetic energy ratio $\Delta\left(K . E_{2} / K . E_{1}\right)$ value for the second regime is 2.8 times greater than the first regime.

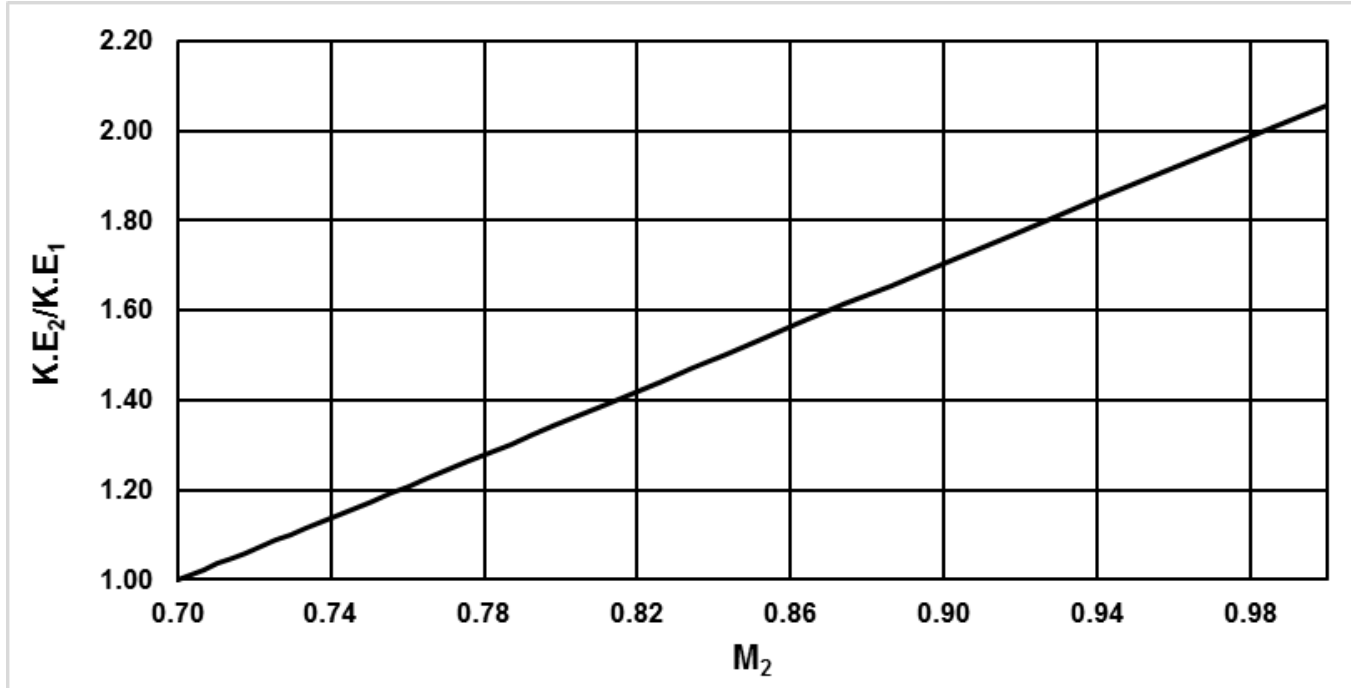

Figure 3.7. Variation of kinetic energy with flow Mach number 
Also, a sharp increase in kinetic energy ratio $\Delta\left(K . E_{2} / K . E_{1}\right)$ is observed in the third regime, which is 2.4 times greater than the previous $\Delta \mathrm{M}_{2}$ (i.e., $\mathrm{M}=0.92$ to $\mathrm{M}=0.94$ ) for the same time interval. It is 4.0 times greater than the average change in kinetic energy ratio $\Delta\left(\mathrm{K} \mathrm{E}_{2} / \mathrm{K} . \mathrm{E}_{1}\right)$ value of the second regime. Quantitatively, the change in kinetic energy ratio value is 0.2011 for the third regime and reaches to the maximum value of kinetic energy ratio K.E. $E_{2} / \mathrm{K}_{1} \mathrm{E}_{1}$ as 2.0563 at sonic Mach number.

Figure 3.8 shows the variation of static temperature to dynamic temperature ratio $T_{2} / T_{d}$ with flow Mach number M. From the plot, it has been observed that there is a continuous decrement in static temperature to dynamic temperature ratio from $\mathrm{M}=0.7$ to $\mathrm{M}=1$. Static temperature to dynamic temperature ratio change has been identified for three different $\Delta \mathrm{M}_{2}$ such as from $\mathrm{M}=0.7$ to $\mathrm{M}=0.845$, from $\mathrm{M}=0.845$ to $\mathrm{M}=0.94$ and from $M=0.94$ to $M=1$. It has been observed that the change in pressure ratio $\Delta\left(T_{2} / T_{d}\right)$ is decreases between $M=0.7$ to $M=0.845$. Afterwards, an increase in the static temperature to dynamic temperature decrement ratio is observed between $\mathrm{M}=0.845$ to $\mathrm{M}=0.94$ and then there is a sudden sharp increase in static temperature to dynamic temperature decrement ratio between $\mathrm{M}=0.94$ to $\mathrm{M}=1$. The average change in static temperature to dynamic temperature ratio $\Delta\left(\mathrm{T}_{2} / \mathrm{T}_{\mathrm{d}}\right)$ values has been calculated as 0.1131 and 0.1999 for first two $\Delta \mathrm{M}_{2}$ respectively. From the calculations, it has been observed that the average change in static temperature to dynamic temperature decrement ratio is almost 1.8 times greater for $0.845<\mathrm{M}<0.94$ when compared to $0.7<\mathrm{M}<0.845$. Between $\mathrm{M}=0.94$ and $\mathrm{M}=1, \Delta\left(\mathrm{T}_{2} / \mathrm{T}_{\mathrm{d}}\right)=0.6319$, which is almost 2.1 times greater in magnitude for the same time step interval (i.e., between $\mathrm{M}=0.92$ and $\mathrm{M}=0.94$ ) and 3.2 times greater in magnitude when compared to the average value of $\Delta\left(\mathrm{T}_{2} / \mathrm{T}_{\mathrm{d}}\right)$ between $\mathrm{M}=0.845$ and $\mathrm{M}=$ 0.94 .

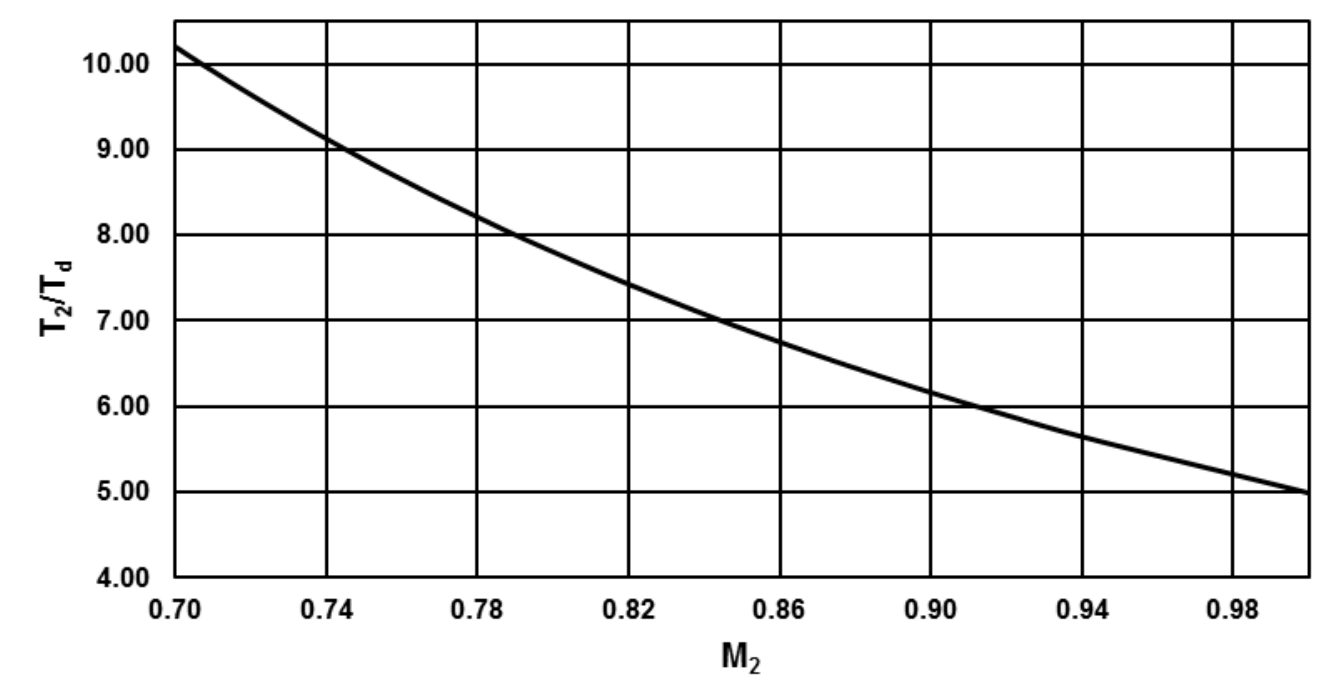

Figure 3.8. Variation of static temperature to dynamic temperature ratio with flow Mach number

From the obtained results, it can be clearly seen that from the static temperature to dynamic temperature ratio - flow Mach number plot, dynamic temperature increases rapidly, once the flow approaches the sonic conditions which means that, there is high dynamic temperature in the flow field near the vicinity of sonic state. The minimum value of static temperature to dynamic temperature ratio $T_{2} / T_{d}$ has been found as 4.9941 with maximum dynamic temperature of $55.11 \mathrm{~K}$ at flow Mach number 1. 


\section{Conclusions}

Unsteady heat addition near the vicinity of sonic conditions has been investigated analytically for compressible subsonic flow between $\mathrm{M}=0.7$ and $\mathrm{M}=1$. The results have been illustrated as the variation of flow properties corresponding to change in flow Mach number during heat addition. From the obtained results, it is obvious that the flow properties change severely near the immediate vicinity of the sonic point.

1. The enthalpy and temperature gradually increase and reaches a maximum value at flow $\mathrm{M}=0.845$ and then smoothly decreases with a minimum incremental change in the magnitude of these flow property till $\mathrm{M}=0.94$. Finally, both enthalpy and temperature suddenly decrease near the sonic regime.

2. Flow velocity and kinetic energy increases slowly between $\mathrm{M}=0.7$ to $\mathrm{M}=$ 0.94 where after there is a sudden acceleration close to the choking point. This investigation provided an insight into sudden variation of flow properties between $\mathrm{M}=0.94$ to sonic point.

3. The obtained results clearly point to sudden acceleration of the subsonic flow as it approaches $M=1$. Increase in unsteadiness in the flow field as flow approaches $M=1$ is also indicated from the results.

4. Based on the above results, it is concluded that the input heat energy was utilized to increase the kinetic energy of the flow thereby reducing the temperature of the flow once the flow approaches the sonic state.

\section{Acknowledgements}

This research did not receive any specific grant from funding agencies in the public, commercial, or not-for-profit sectors.

\section{References}

[1] H. Shapiro, The Dynamics and Thermodynamics of Compressible Fluid Flow, Ronald Press, New York, (1953), pp. 129-261.

[2] P. A. Thompson, Compressible Fluid Dynamics, McGraw-Hill, New York, (1972), pp. 374-437.

[3] M. A. Saad, Compressible Fluid Flow, Prentice Hall, Inc., Upper Saddle River, NJ, (1985), pp. 226-291.

[4] Z. Y. Guo, Thermally Induced Effects on Fluid Flow, Annual Review of Heat Transfer, (1994);1, 207-270.

[5] P. J. Villey, Les Ecoulements Lineaires des Gaz Parfaits, Journal de Physique et le Radium, (1942);32 (4), 79-80.

[6] Szczeniowsky, Flow of Gas Through a Tube of Constant Cross- Section with Heat Exchange, Canadian Journal of Research, (1945); 23 (1), 1-11.

[7] Kahane, L Lees, Unsteady One-Dimensional Flows with Heat Addition or Entropy Gradients, Symposium on Combustion and Flame and Explosion Phenomena, (1949); 32 (1), 222-229.

[8] A. M. Abourabia, T.Z. Abdel Wahid, The unsteady Boltzmann kinetic equation and non-equilibrium thermodynamics of an electron gas for the Rayleigh flow problem, Canadian Journal of Physics, (2010); 88, 501-511.

[9] T. Zakaraia, W. Wahid, Kinetic and Thermodynamic Treatment for the Exact Solution of the Unsteady Rayleigh Flow Problem of a Rarefied Homogeneous Charged Gas, Journal of Non-Equilibrium Thermodynamics, (2012); 37 (2), 119-141.

[10] A. Bandyopadhyay, A. Majumdar, Modeling of Compressible Flow with Friction and Heat Transfer Using the Generalized Fluid System Simulation Program, In: Proceedings of the Thermal Fluid Analysis Workshop, NASA 20070036728, 
Cleveland, OH, (2007);1-14.

[11] P. Balachandran, Gas Dynamics for Engineers, PHI Learning, New Delhi, India, (2010); pp. 155-168.

[12] Rajarshi Das, Heuy-Dong Kim, Unsteady Rayleigh Flow at Transonic Speeds, Journal of Propulsion and Power, American Institute of Aeronautics and Astronautics (AIAA), (2016); 1-4. 